\title{
Acute Sleep Loss Upregulates the Synaptic Scaffolding Protein, Homer1a, in Non-canonical Sleep/Wake Brain Regions, Claustrum, Piriform and Cingulate Cortices
}

\author{
Jingxu Zhu', Jennifer Hafycz', Brendan T. Keenan, Xiaofeng Guo, Allan Pack and \\ Nirinjini Naidoo*
}

Division of Sleep Medicine, Perelman School of Medicine, University of Pennsylvania, Philadelphia, PA, United States

OPEN ACCESS

Edited by:

Christelle Peyron,

INSERM U1028 Centre de Recherche en Neurosciences de Lyon, France

Reviewed by:

Radhika Basheer,

Harvard Medical School,

United States

Michele Bellesi,

University of Bristol, United Kingdom

${ }^{*}$ Correspondence:

Nirinjini Naidoo

naidoo@pennmedicine.upenn.edu

${ }^{t}$ These authors share first authorship

Specialty section:

This article was submitted to Sleep and Circadian Rhythms,

a section of the journal

Frontiers in Neuroscience

Received: 03 October 2019

Accepted: 20 February 2020

Published: 13 March 2020

Citation:

Zhu J, Hafycz J, Keenan BT,

Guo X, Pack A and Naidoo N (2020)

Acute Sleep Loss Upregulates

the Synaptic Scaffolding Protein,

Homer1a, in Non-canonical

Sleep/Wake Brain Regions,

Claustrum, Piriform and Cingulate

Cortices. Front. Neurosci. 14:188.

doi: 10.3389/fnins.2020.00188
Homer proteins are a component of the post-synaptic density of neurons that are necessary for the maintenance and consolidation of behavioral state. The dominant negative protein homer1a is rapidly increased by neuronal activity and sleep loss. Homer1a knockout mice with globally absent homer1a have reduced ability to sustain wakefulness during the active period. It is not known whether homer1a is required globally or in very specific brain regions or neurons for its role in maintaining wake. In this study, we examined the expression of homer1a, an immediate early gene involved in intracellular signaling cascades, in mice subjected to extended wakefulness. We found that mice displayed increased expression of homer1a in the claustrum, a brain region thought to be involved in consciousness, as well as the cingulate and piriform cortices compared to non-sleep deprived mice. In situ hybridization (ISH) studies also indicate that homer1a is not induced in the known wake promoting regions with sleep deprivation, but is instead upregulated primarily in the claustrum and piriform cortex. Examination of homer1a expression levels with recovery sleep after sleep deprivation indicate that baseline homer1a expression levels were restored. Further, we have identified that homer1a is upregulated in excitatory neurons of the claustrum suggesting that homer1a promotes wakefulness through activating excitatory neurons. This work identifies regions previously unknown to be involved in sleep regulation that respond to acute sleep deprivation or enhanced waking.

Keywords: claustrum, homer1a, sleep deprivation, excitability, in situ hybridization

\section{INTRODUCTION}

Homer proteins function at the post-synaptic density as scaffolds, where they link several molecules important for cellular signaling. Specifically, homer1 functions as an adaptor to metabotropic glutamate receptors (mGluRs) as well as Shank proteins, PSD-95 expressed on NMDA receptors, and $\mathrm{IP}_{3}$ receptors expressed on the endoplasmic reticulum (Paschen and Mengesdorf, 2003; 
Piers et al., 2012). Homer1, therefore, has a role in synaptic plasticity and intracellular calcium signaling. Homer1 has three isoforms in mammals, homerla, homer1b, and homer1c. Homerla is classified as an immediate early gene and is the short form of homer1, lacking the C-terminal coiled coil domain, while homer $1 \mathrm{~b}$ and homer1c are long forms. Homerla competes with the long forms of homer1 in a dominant negative manner, disrupting the signaling connections between homer1b, homer1c, and their binding partners.

Homerla is a known molecular correlate of sleep loss (Nelson et al., 2004; Maret et al., 2007; Mackiewicz et al., 2008; Naidoo et al., 2012). Homer1a mRNA is upregulated under sleepdeprived conditions in mouse cortices and hypothalamic tissue (Nelson et al., 2004; Mackiewicz et al., 2007; Maret et al., 2007). We have previously reported that global knockout of homerla in mice leads to an inability to maintain long bouts of wakefulness, suggesting a role for homerla in maintenance of wakefulness (Naidoo et al., 2012). Further, it has been shown that homerla is required for the alteration of synapses during sleep (Hu et al., 2010). More recent data indicate that homerla protein moves into the synapse during sleep and is responsible for synaptic downscaling (Diering et al., 2017). This evidence suggests that homerla function is crucial for proper sleep-wake behaviors and synaptic homeostasis. Despite this, little is known about the direct role of homerla on sleep and wake behavior or its mechanism of action.

In order to better understand the role of homerla as an immediate early gene product in sleep and wake, we used in situ hybridization to assay homerla mRNA expression across the mouse brain under conditions of acute sleep loss compared to that in sleeping mice. Previous studies have examined homerla expression following six or more hours of sleep deprivation. We report in this study that homerla is not robustly induced in the known wake promoting regions following three or less hours of sleep loss, but is instead upregulated primarily in the claustrum, piriform and cingulate cortices.

In addition, following recovery sleep homerla expression levels in these neuronal groups are restored to baseline levels. Finally, we have found that sleep-loss induced homer1a expression in claustral neurons co-localizes with that of CAMKII $\alpha$, a marker for excitatory neurons. Together, these results identify the claustrum as a novel brain region that demonstrates changes in homer1a expression in response to very short periods of sleep deprivation (SD) and could therefore be involved in the regulation of sleep and wake.

\section{MATERIALS AND METHODS}

\section{Mice}

All experiments were performed on male mice. C57/BL6 mice were 8 weeks of age \pm 1 week. All mice were maintained on $12 \mathrm{hr}$ light/dark cycle in a sound attenuated recording room, temperature $22-24^{\circ} \mathrm{C}$. Food and water were available ad libitum. Animals were acclimated to these conditions for 7 days before beginning any studies. All animal experiments were performed in accordance with the guidelines published in the NIH Guide for the Care and Use of Laboratory Animals and were approved by the University of Pennsylvania Animal Care and Use Committee.

\section{Sleep Deprivation}

Mice were sleep deprived (SD) for either $1 \mathrm{~h}$ or 3 hours. Deprivation was initiated at $12 \mathrm{pm}$ (ZT5) for $1 \mathrm{~h}$ or 10 am (ZT 3) for $3 \mathrm{~h}$. Deprivations were performed through gentle handling following a 5 day acclimatization period for handling procedures as previously described (Naidoo et al., 2008). Undisturbed spontaneously sleeping mice were used as diurnal controls at $1 \mathrm{pm}$ (SC).

\section{Recovery Sleep}

Since 3-hour sleep deprivation produced the greatest change in Homer1a expression only mice that underwent 3-hour sleep deprivation, (10 am-1 pm) were allowed 3-hour recovery sleep (1 pm-4 pm). Mice were sacrificed at the end of the recovery sleep (RS) period. Diurnal undisturbed sleeping controls were obtained at $4 \mathrm{pm}$.

\section{Tissue Collection}

The solutions used for in situ hybridization (ISH) were prepared using $0.1 \%$ diethylpyrocarbonate (DEPC) water. Mice were perfused transcardially with $0.9 \%$ saline, followed by $4 \%$ paraformaldehyde immediately following undisturbed sleep, sleep deprivation or the recovery sleep period. Brains were collected and post-fixed in $4 \%$ paraformaldehyde solution with RNase inhibitor at $4^{\circ} \mathrm{C}$. After $24 \mathrm{~h}$ brains were moved to $30 \%$ sucrose with RNase inhibitor at $4^{\circ} \mathrm{C}$. Brains were sectioned coronally in a cryotome at 40 micrometers, collected in 1:6 series with free-floating solution (1xPBS with $0.05 \%$ and RNase inhibitor), and stored at $4^{\circ} \mathrm{C}$ until processed for ISH, RNAscope or IHC. A complete set of brain sections representing the entire brain were used.

\section{Digoxigenin (DIG) Labeled Probes in situ Hybridizations Procedure}

Sense and antisense DIG-labeled RNA probes were respectively synthesized from full length cDNA clones of Homerla (or Galanin for studies of VLPO (cDNA clone MGC:54666 IMAGE:6476459 plasmid, Invitrogen) using T7 (anti-sense) and SP6 (sense) polymerases (Roche). The probes were stocked at $-80^{\circ} \mathrm{C}$ until used for hybridization. All procedures were performed at room temperature unless a different temperature was stated. Free-floating coronal sections were fixed with $4 \%$ PFD for $30 \mathrm{~min}$ and then permeabilized with $0.4 \%$ TritonX100 overnight. The sections were incubated with $0.1 \mathrm{M}$ triethanolamine and $0.25 \%$ acetic anhydride for $10 \mathrm{~min}$, and then rinsed with $2 \mathrm{X}$ saline-sodium citrate (SSC) solution. Sense and antisense probes were denatured at $85^{\circ} \mathrm{C}$ for $3 \mathrm{~min}$ and then on ice for $5 \mathrm{~min}$ before mixed with hybridization buffer $(1: 1000)$ with $0.25 \mathrm{mg} / \mathrm{ml}$ tRNA (Roche) to make hybridization solutions. The sections were hybridized with hybridization solutions for 16-18 hours overnight at $55^{\circ} \mathrm{C}$ in a humidified chamber. The sections were washed with $4 \times, 2 \times$, and $1 \times$ SSC solution each for $30 \mathrm{~min}$ with three time changing and $0.1 \mathrm{X}$ SSC solution at 
$60^{\circ} \mathrm{C}$ for $1 \mathrm{~h}$. To detect the signals, the sections were incubated with anti-digoxigenin-AP antibody (1:3000, Roche 11093274910) for $48 \mathrm{~h}$ at $4^{\circ} \mathrm{C}$ and then developed with NBT/BCIP (1:100, Sigma) for $16-18 \mathrm{~h}$ at $4^{\circ} \mathrm{C}$. The sections were mounted using an aqueous mounting solution - Vecte Mount AQ (Vector, H5501). Some sections were counterstained with GNG2 (Abcam, ab19825) antibody (see below IHC) before mounting to examine the colocalization of Homer1a and GNG2 at claustrum. Digital brightfield images were obtained on a Leica microscope (Leica).

\section{Quantitative Analysis of Dioxigenin (DIG) ISH Images}

To compare the ISH changes of homerla after sleep deprivation and recovery sleep, three groups of animals (SD, SC, and SR) that were treated equally for perfusion, cutting and DIG ISH were used. Sections from a 1 in 12 series through the claustrum between Bregma 1.4 to $0.2 \mathrm{~mm}$ were used for DIG ISH. Three sections from the claustrum and piriform cortex were analyzed per animal. All images were captured using the $20 \times$ objective on a Leica DM5500 microscope at the same setting. Calculation of the percent area occupied by Homerla ISH positive area fraction was performed using NIH ImageJ (National Institutes of Health, Bethesda, MD, United States) software domain (Papadopulos et al., 2007). To analyze Homer1a expression on the images, $\mathrm{NIH}$ ImageJ (National Institutes of Health, Bethesda, MD, United States) software domain was used (Papadopulos et al., 2007). Briefly, every individual color image was converted to 8-bit format image with the Image/Type/8-bit command. The 8-bit gray scale image was adjusted with the Image/Adjust/Threshold Command for threshold 120. From Analyze/Measure command, the percentage of total area that the positive homerla ISH occupies was automatically displayed on the result sheet. These data were used for statistical analyses.

\section{RNAscope in situ Hybridization (ISH)}

RNAscope in situ hybridization was performed in $40 \mu \mathrm{m}$ floating brain tissue sections using the method modified from Grabinski et al. (2015) to detect and visualize the co-localization of Homerla with CamKII $\alpha$, GAD1 or Gng2. The RNAscope Multiplex Fluorescent Reagent Kit (323100) and RNAscope probes [mouse Homer1a (432441-C2), Gng2 (462481), GAD1 (400951-C3), CamKII $\alpha$ (445231)] were from Advanced Cell Diagnostics (ACD, Hayward, CA, United States). For the modified manual RNAscope experiments free-floating coronal sections were fixed with $4 \%$ PFD for $30 \mathrm{~min}$. After washing with PBS, the sections were treated with $3 \% \mathrm{H}_{2} \mathrm{O}_{2}$ for $30 \mathrm{~min}$ and then permeabilized with $0.4 \%$ Triton-X100 overnight. Sections were mounted on superfrost plus slides (Fisher Scientific) and dried at $60^{\circ} \mathrm{C}$ for $30 \mathrm{~min}$. After cooling to room temperature, the slides were pretreated with III reagent for $20 \mathrm{~min}$ at $40^{\circ} \mathrm{C}$. Hybridization with double probes (Gng-C1 and Homer1a-C2) or triple probes (CanKII $\alpha-\mathrm{C} 1$, Homer1a-C2, and GAD1-C3) were performed for 2 hours at $40^{\circ} \mathrm{C}$, followed by amplification. TSA fluorophores labeling was carried out using TSA Fluorescein for $\mathrm{C} 1, \mathrm{TSA} \mathrm{Cy} 3$ for C2 and TSA Cy5 for C3 respectively. Fluorescent images were captured using a Leica confocal microscope (SP5 AOBS, Leica).

\section{Immunohistochemistry (IHC) and Immunofluorescence (IF) Staining Procedures}

To identify different known wake-active neurons, IHC was performed using a commercially available kit according to manufacturer's instructions (ABC Immunodetection kit, Vector Laboratories, Burligame, CA, United States) for Orexin-A (1:1000, sc-8070) in the lateral hypothalamus, Tyrosine Hydroxylase (TH, 1:1000, AB152, Millipore) in locus coeruleus. Histidine Decarboxylase (HDC, 1:2000, 2263B260-1, EuroProxima) in tuberomammillary nucleus (TMN). Briefly, $40 \mu \mathrm{m}$ brain sections were treated with $0.3 \% \mathrm{H}_{2} \mathrm{O}_{2}$ in PBS for 30 minutes and then blocked with $0.4 \%$ normal donkey serum (NDS) and 1\% BSA for $2 \mathrm{~h}$. The primary antibody was applied overnight at room temperature washed and then incubated with biotinylated secondary antibody for $60 \mathrm{~min}$. The reaction was developed with an avidin-biotin complex reaction and the signals were detected by DAB. Digital brightfield images were recorded on a Leica microscope (Leica).

Double immunofluorescence staining (Naidoo et al., 2011) was used to characterize the waking c-Fos response in claustrum, orexinergic neurons and LC. Latexin (1:100, PA528534, Invitrogen), Orexin-A and TH were labeled with Alexa fluor 594 (Invitrogen) by secondary antibodies and c-Fos (1:1000, ab208942, Abcam) was labeled with Alexa fluor 488 (Invitrogen) by secondary antibody. Fluorescent images were captured using a Leica confocal microscope (SP-5 AOBS, Leica).

\section{Punches and Q-PCR}

For these experiments, mice were euthanized by cervical dislocation and brains were quickly removed, rapidly frozen on dry ice, and stored at $-80^{\circ} \mathrm{C}$. Micropunches of the brain were performed in a cryostat as described (Palkovits, 1983; Palkovits et al., 1985). Five brain regions, the claustrum, piriform cortex, lateral hypothalamus, tuberomammillary nucleus, and locus coeruleus, were dissected according to the mouse brain atlas from Allen Institute for Brain Science (Bakker et al., 2015; Lein et al., 2007). Claustrum (CLA) was dissected using $1.2 \mathrm{~mm}$ i.d. Harris Uni-Core Micro-Punch (Whatman) from four $300 \mu \mathrm{m}$ sections between approximately 1.6 to $0.4 \mathrm{~mm}$ Bregma. Piriform cortex (PIR) was dissected from four $300 \mu \mathrm{m}$ sections between approximately 0.4 to $-0.8 \mathrm{~mm}$ Bregma using $1.2 \mathrm{~mm}$ i.d. micropunch. Lateral hypothalamus (LHA) was dissected from four $300 \mu \mathrm{m}$ sections between approximately -0.8 to $-2.0 \mathrm{~mm}$ Bregma using $1.2 \mathrm{~mm}$ i.d. micropunch. Tuberomammillary nucleus (TMN) was dissected from two $300 \mu \mathrm{m}$ sections between approximately -2.0 to $-2.6 \mathrm{~mm}$ Bregma using $1.2 \mathrm{~mm}$ i.d. micropunch. Locus coeruleus (LC) was dissected from two $200 \mu \mathrm{m}$ sections between approximately -5.2 to $-5.6 \mathrm{~mm}$ Bregma using $1.0 \mathrm{~mm}$ i.d. micropunch.

RNA extraction was performed using RNAqueous-Micro Total RNA Isolation Kit (Invitrogen) and on-column DNase digestion was performed with RNase-Free DNase Set (Qiagen). RNA concentration and purity were measured by Nanodrop 1000 spectrophotometer. Thirty-six samples were randomly chosen from the five regions for evaluation of RNA integrity (RIN) 
using Agilent 2100 Bioanalyzer RNA 6000 Nano chip. All samples measured had a RIN value above 8.0.

cDNA was synthesized from RNA using High-Capacity RNA-to-cDNA kit (Applied Biosystems), and quantitative real-time PCR assays were performed using TaqMan assays for the following gene targets: Fos (Mm00487425_m1), Arc (Mm00479619_g1), BDNF (Mm01334043_m1), Homer1 (Mm00516275_m1), Homer1a (customized AIT95T0, derived from nucleotides 1342-2140); region specific markers: Gng2 (Mm00726459_s1) (marker for CLA), Hcrt (Mm01964030_s1) (marker for LHA), Hdt (Mm00456104_m1) (marker for TMN), Th (Mm00447557_m1) (marker for LC); house-keeping genes Hprt (Mm01545399_m1), and Tbp (Mm00446971_m1). RT-PCR reactions were performed using an Applied Systems 7900HT Fast Real-Time PCR System with 10ng of cDNA in each reaction. For the gene assays that may detect genomic DNA, namely Arc, Homerla, Gng2 and Hcrt, no-RT control experiment (reverse transcriptase was removed from the cDNA synthesis reaction to detect any residual amplification from genome DNA template) was run for each sample to confirm the effectiveness of DNase-treatment. Relative gene expression was calculated using the deltaCt method (Vandesompele et al., 2002; Schmittgen and Livak, 2008), where the Ct value of each target gene was corrected using the geometric mean of the $\mathrm{Ct}$ values of the two housekeeping genes. Analyses were performed on negative deltaCt $(-\Delta \mathrm{Ct})$ values, such that more positive values indicate higher expression.

\section{STATISTICAL METHODS}

\section{q-PCR Analyses and Statistics}

To evaluate differences across conditions, we performed a joint hypothesis test simultaneously assessing whether there were any differences in PCR values across three a priori pairwise comparisons of interest: (i) sleep deprivation vs. 3-hour spontaneous sleep control, (ii) sleep recovery vs. 6-hour control, and (iii) sleep deprivation vs. sleep recovery. This test is similar to an ANOVA, providing an overall comparison of $>2$ groups, but allows for separate control groups. Given multiple genes in each region, statistical significance of the joint hypothesis test was based on a Hochberg corrected threshold (Hochberg, 1988). If the joint null hypothesis was rejected, we subsequently evaluated each pairwise comparison to determine which differences were driving the overall effects, again with a Hochberg correction for multiple comparisons (Hochberg, 1988; Huang and Hsu, 1994). Results are presented as model-estimated means and $95 \%$ confidence intervals within each group.

\section{In situ Hybridization (ISH) Analysis}

Prior to analysis, average values were obtained within each section and region, resulting in 3 separate measurements in the claustrum or piriform cortex for each mouse. Given this, comparisons of percentage area among conditions were performed using a mixed model analysis of variance (ANOVA) with a random intercept term to account for multiple measures per animal. This analysis assessed the global null hypothesis of no differences among conditions (control, sleep deprivation, recovery sleep). If this global null hypothesis was rejected $(p<0.05)$, we performed subsequent pairwise comparisons between conditions; statistical significance in pairwise comparisons was based on a Hochberg corrected threshold (Hochberg, 1988). Percentage area values were natural log transformed for analyses in order to satisfy parametric assumptions; for interpretability, results are presented as groupspecific geometric means and $95 \%$ confidence intervals.

\section{RESULTS}

\section{Acute Sleep Loss Induces Homer1a Expression in the Claustrum, Cingulate and Piriform Cortices}

We have previously reported that homerla mRNA expression is increased in the brain with $6 \mathrm{~h}$ of sleep loss (Mackiewicz et al., 2007) and that homerla is required for the maintenance of wakefulness (Naidoo et al., 2012). To examine the spatial distribution of changes in expression of homerla mRNA in the brain following sleep loss, mice at two months of age were subjected to either one or three hours of sleep deprivation beginning at ZT 5 (noon) or ZT 3 (10 am), respectively. Thus, sleep deprivation ended at the same diurnal time in both groups. With this acute sleep deprivation paradigm, we were able to assess more immediate molecular changes that occur with sleep loss, particularly in the form of homerla as an immediate early gene product. Mice were perfused immediately following sleep deprivation and brain slices were subjected to in situ hybridization for the Homerla gene. Diurnal sleeping controls were perfused at the same time points.

Overall, homerla mRNA expression is increased following sleep deprivation in the claustrum, cingulate, and piriform cortices as revealed by in situ hybridization (Figure 1). The changes in expression levels of homer1a mRNA were more robust in these same regions and in the motor cortex following three-hours of sleep deprivation; the highest expression was localized to the claustrum and the piriform cortex (Figure 1). Further, immunostaining of Gng2, a protein enriched in the claustrum was used as a claustral marker (Wang et al., 2017), coupled with in situ hybridization to homerla in matched brain sections, confirmed localization of homerla expression to the claustrum (Figure 2E). As the Gng2 antibody is not particularly robust, since clearly immunostained puncta are not observed, we also confirmed homerla localization to the claustrum using RNAscope double in situ hybridization with mRNA for Gng2 (Figure 2). Here we found that the homerla and Gng2 mRNA probes co-localize to the same neurons (Figure 2F).

\section{Homer1a Expression Is Localized to Excitatory Neurons in the Claustrum}

While the majority of claustrum neurons are excitatory, the region consists of a heterogeneous population of both excitatory and inhibitory neurons. To determine if there was differential expression of homerla in a subpopulation of claustrum 


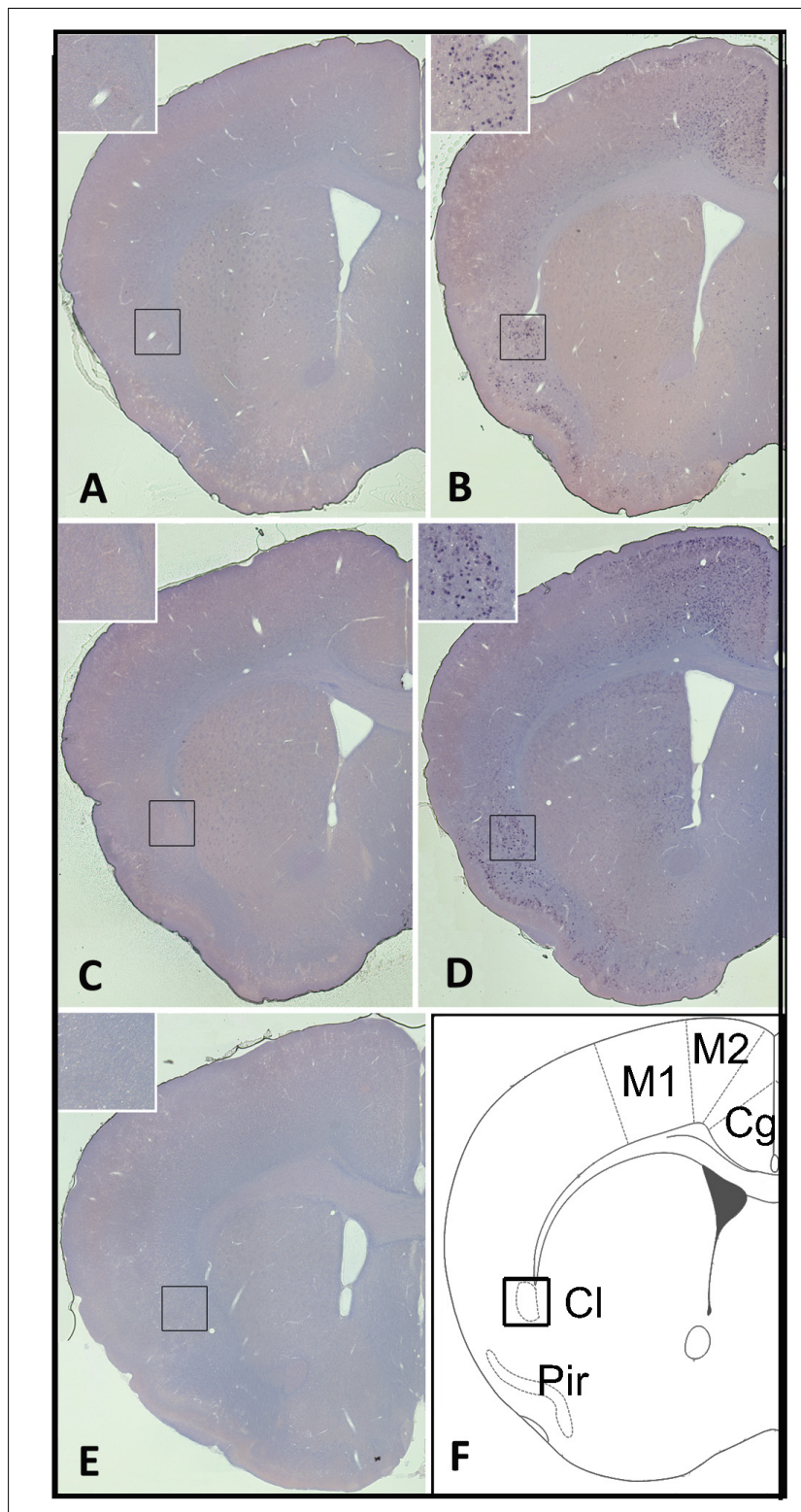

FIGURE 1 | Sleep loss induces homer1a expression in the claustrum, cingulate and piriform cortices. (A) Homer1a expression levels in $1 \mathrm{hr}$ sleep control claustrum enlarged; (B) Homer1a expression levels following $1 \mathrm{~h}$ sleep deprivation (SD), claustrum enlarged; (C) Homer1a expression levels in $3 \mathrm{~h}$ sleep diurnal control, claustrum enlarged; (D) Homer1a expression levels following 3 h SD; (E) In situ hybridization sense control; (F) diagram of brain slice showing pertinent regions. Bar size $1 \mathrm{~mm}$.

neurons, we carried out in situ hybridization for homerla and co-immunostaining with either CAMKII $\alpha$ or GAD67 as markers for excitatory and inhibitory neurons, respectively. Our immunostaining revealed that the increased homerla protein expression in sleep-deprived mice co-localizes with excitatory CAMKII $\alpha$-positive cells (Figure 3). This suggests that the expression of homerla with extended wakefulness could influence cellular excitability.

\section{Homer1a Expression Is Not Induced in Canonical Sleep/Wake Brain Regions Following Sleep Deprivation}

Interestingly, there were no apparent changes in homerla expression by in situ hybridization in classically studied wakepromoting and sleep-promoting brain regions, including the noradrenergic neurons of the locus coeruleus (LC), orexin neurons of the lateral hypothalamus (LHA), the histaminergic neurons in the tuberomammillary nucleus (TMN), or the sleep promoting ventrolateral pre-optic area (VLPO) (Figure 4). To verify that the traditional wake active neuronal populations were in fact activated during enforced wakefulness/sleep deprivation we carried out c-Fos immunostaining and found that c-Fos was expressed with $3 \mathrm{~h}$ of sleep deprivation in the locus coeruleus, orexin neurons in the LHA and histaminergic neurons in the TMN, but there was no increase in homer 1a expression (see Figure 5).

\section{Homer1a Expression Is Reduced With Recovery Sleep}

In order to determine if homerla mRNA expression is downregulated with sleep, we subjected mice to $3 \mathrm{~h}$ of sleep deprivation followed by a 3-hour period of recovery sleep. These sleepdeprived mice were perfused immediately following $3 \mathrm{~h}$ of recovery sleep. There was a separate group of normally sleeping mice that were sacrificed at the same diurnal time. Homerla expression levels in mice that had been allowed recovery sleep were similar to homer1a expression levels in the undisturbed diurnal spontaneously sleeping control mice in the brain regions of interest (motor cortices, piriform cortices, claustrum) as determined by in situ hybridization (see Figure 6).

Quantification of the homer1a in situ hybridization signal in the claustrum and piriform cortex indicate that homerla mRNA is significantly altered with behavioral state in these regions (see Supplementary Table S1). Specifically, homer $1 a$ in the claustrum of young mice is increased with sleep deprivation compared to undisturbed sleep $(1.91[0.69,5.23]$ vs. $0.27[0.10,0.75] \%$ area; $p=0.007)$ and decreased with recovery sleep compared to sleep deprivation $(0.34[0.12,0.92]$ vs. $1.91[0.69,5.23] \%$ area; $p=0.017)$. There was no difference in homerla during undisturbed sleep and recovery sleep in the claustrum $(p=0.780)$. In the piriform of these young mice, we again observed increased homer 1 a during sleep deprivation compared to undisturbed sleep (5.79 $[2.48,13.53]$ vs. $0.44[0.19,1.03] \%$ area; $p<0.0001)$. While there is trending evidence of a reduction in values during recovery sleep compared to sleep deprivation $(1.85[0.79,4.33]$ vs. $5.79[2.48,13.53] \%$ area; $p=0.063)$, homer $1 a$ expression was still elevated in the piriform following recovery sleep compared to undisturbed sleep $(1.85[0.79,4.33]$ vs. $0.44[0.19,1.03] \%$ area; $p=0.019$ ).

\section{Homer1a Expression in Mice Following Sleep Loss Is Recapitulated by q-PCR}

Changes in homerla expression with sleep loss were also confirmed by quantitative PCR using punches of the key regions identified by in situ hybridization. 


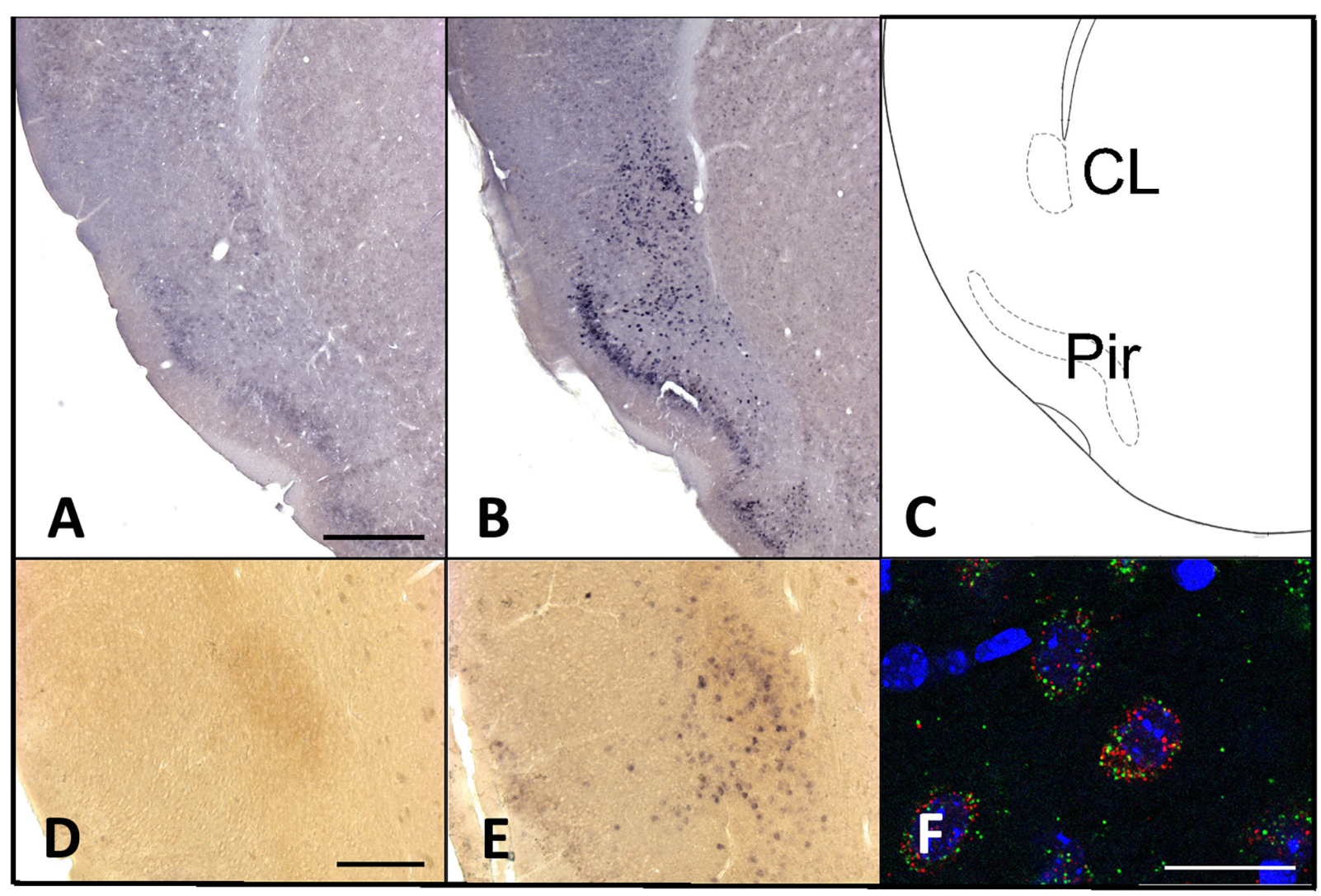

FIGURE 2 | Increases in homer1a mRNA are localized to the claustrum and piriform cortex. (A) Homer1a in situ 3 h sleep control (SC); (B) Homer1a in situ hybridization $3 \mathrm{~h}$ sleep deprivation (SD); (C) corresponding zoomed-in atlas image; (D) Homer1a in situ and GNG2 immunostain in $3 \mathrm{~h}$ sleep control; (E) Homer1a in situ and GNG2 immunostain in 3 h SD; (F) Homer1a (red), and Gng2 (green) RNAscope with DAPI (blue). Bar size A: $500 \mu m$, D: $250 \mu m$, F: $25 \mu m$.

As a first step, we directly examined differences in homerla expression levels via qPCR across the different regions during undisturbed sleep (Figure 7A and Supplementary Table S2). We observed significant differences across regions $(p<0.0001)$; homerla expression in the claustrum and piriform cortex was significantly greater than expression in other canonical wake regions ( $\mathrm{LH}, \mathrm{TMN}, \mathrm{LC})$.

When examining the effects of sleep loss, as for in situ hybridization in Figure 1, homer1a transcript assessed by qPCR is significantly increased with $3 \mathrm{~h}$ of sleep deprivation compared to undisturbed sleep in the claustrum $(-3.54[-3.97,-3.12]$ vs. $-4.83[-5.29,-4.36]-\Delta \mathrm{CT} ; p=0.0004)$ and piriform cortex $(-3.05[-3.36,-2.75]$ vs. $-4.88[-5.19,-4.58]-\Delta \mathrm{CT}$; $p<0.0001$ ) (see Figure 7B and Supplementary Table S3]. To ensure that we had successfully punched out the claustrum, we assayed for transcript levels of Gng2, a marker for the claustrum and determined that Gng2 did not change with behavioral state (Supplementary Figure S1). Homer1a was not increased in the LC or TMN by $\mathrm{qPCR}$, recapitulating the in situ hybridization observation $(p=0.759$ and $p=0.137$, respectively). On the other hand, $\mathrm{qPCR}$ reveals a small, but significant increase in homer1a expression in the LHA with sleep deprivation compared to 3 -hour sleep controls $(-6.87[-7.23$, $-6.51]$ vs. $-7.78[-8.14,-7.42]-\Delta \mathrm{CT} ; p=0.0013$; Figure 7 and Supplementary Tables S2, S3). When examining statistical evidence for differential homer1a responses to sleep deprivation across these regions (Supplementary Table S3), there was a significant interaction ( $p<0.001$; Supplementary Table S3). Increases in homerla with sleep deprivation were generally larger in the claustrum and piriform cortex than canonical wake regions.

We further confirmed our in situ hybridization findings of reduced homerla expression in the claustrum following recovery sleep. In both the claustrum and piriform cortex, homer1a values were significantly reduced after recovery sleep when compared to values during sleep deprivation, and values return to levels seen in control animals sleeping undisturbed for 6 h (see Supplementary Table S3 and Figure 7B). Supporting the differential responses to sleep loss and recovery, statistical interaction tests show regional differences in the comparisons between recovery sleep and sleep deprivation $(p \leq 0.002)$ (Supplementary Table S3).

\section{QPCR Shows Increases in c-fos Following Sleep Deprivation in All Wake-Active Neurons}

Similarly, we utilized qPCR to evaluate $c$-fos expression in wake active brain regions (LC, TMN, lateral hypothalamus) 


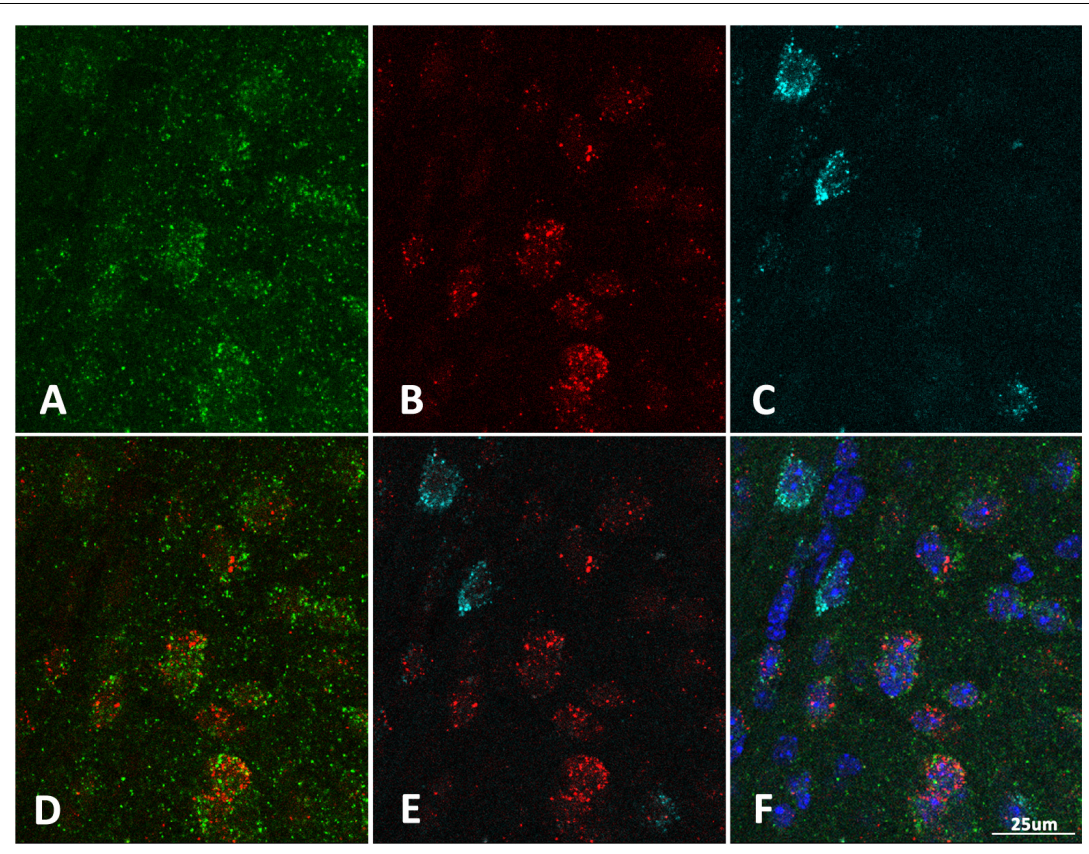

FIGURE 3 | Homer1a expression is localized to excitatory neurons in the claustrum. (A) Immunostain for CAMKIl $(\mathrm{green})$; (B) Immunostain for Homer1a (red); (C) Immunostain for GAD67 (cyan); (D) CAMKIl $\alpha$ and Homer1a overlap; (E) GAD67 and Homer1a merge; (F) Merge CAMKIll $\alpha$, GAD67, Homer1a, and DAPI (blue). Bar size: $25 \mu \mathrm{m}$.

as well as in the claustrum and piriform cortex following sleep deprivation and sleep recovery. In contrast to results for homer1a, $c$-fos was expressed at similar levels across interrogated brain regions $(p=0.082$; Figures $7 \mathrm{C}, \mathrm{D}$, see Supplementary Table S4).

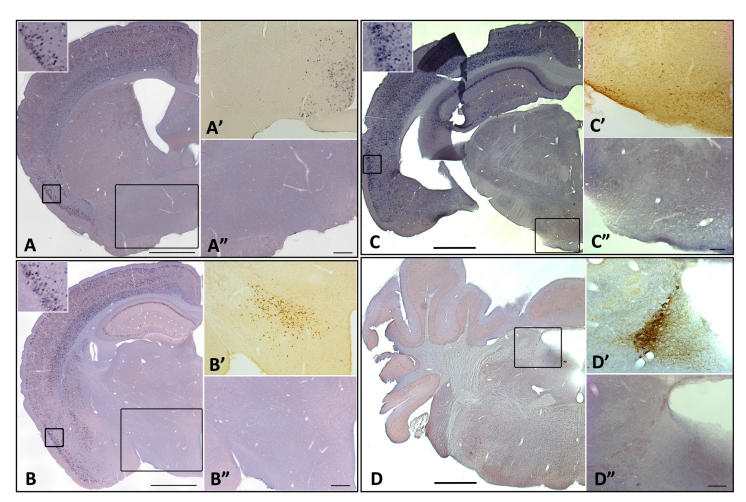

FIGURE 4 | Homer1a expression is not induced in canonical sleep/wake brain regions following sleep deprivation. Homer1a ISH shown as positive control in small inset for each section. Brain sections illustrating. (A) Ventrolateral preoptic nucleus, VLPO, (large inset); ( $\left.\mathbf{A}^{\prime}\right)$ Galanin ISH as a marker for the VLPO; (A") Homer1a in situ in VLPO; (B) lateral hypothalamus, LHA, (large box); (B') Orexin immunostain as a marker for the LHA; (B") Homer1a in situ in enlarged LHA; (C) Tuberomamillary nucleus, TMN, (large box); (C') Histamine immunostain as a marker for the TMN; (C") Homer1a in situ in enlarged TMN; (D) Locus coeruleus, LC, (large box); (D') Tyrosine Hydroxylase immunostain as marker for the LC; (D") Homer1a in situ in enlarged LC. Bar size A,B,C,D: 1 mm, A",B": $250 \mu \mathrm{m}, \mathbf{C}^{\text {,,D": }} 100 \mu \mathrm{m}$.
When examining differences across behavioral conditions, we observed significant increases in $c$-fos with sleep deprivation in all brain regions (see Supplementary Table S4 and Figures 7C,D). While all regions showed increased $c$-fos with sleep loss compared to undisturbed sleep, there were significant regional differences

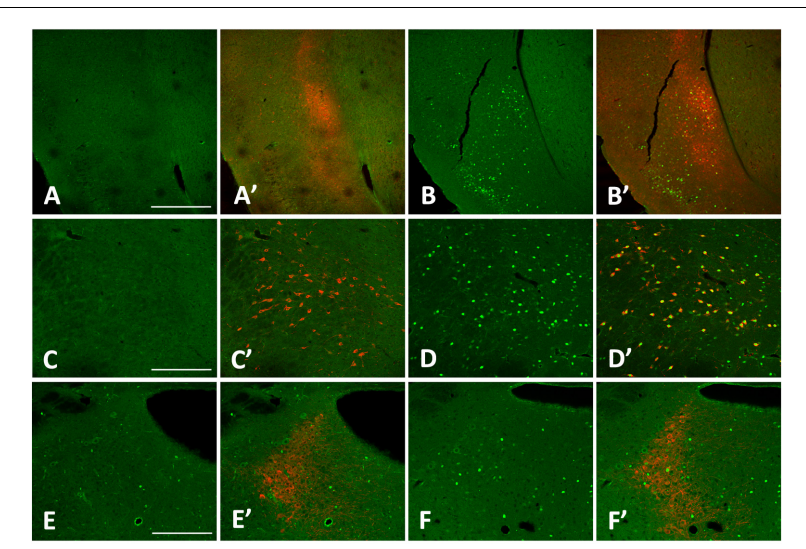

FIGURE 5 | cFos levels increase in the claustrum, lateral hypothalamus (LHA), and locus coeruleus (LC) with 3 h sleep deprivation (SD) compared to sleeping control (SC) animals. (A) 3 h SC cFos staining; (A') 3 h sleep control with cFos and Latexin as marker for the claustrum; (B) $3 \mathrm{~h}$ SD cFos staining; (B') $3 \mathrm{~h}$ SD with cFos and Latexin; (C) 3 h SC cFos staining; (C') 3 h sleep control with cFos and Orexin as marker for the LHA; (D) 3 h SD cFos staining; (D') 3 h SD with cFos and Orexin; (E) 3 h SC cFos staining; (E') 3 h sleep control with cFos and tyrosine hydroxylase (TH) as marker for the LC; F) 3 h SD cFos staining; (F') 3 h SD with cFos and TH. Bar size A: $500 \mu \mathrm{m} ; \mathbf{C , E}: 250 \mu \mathrm{m}$. 


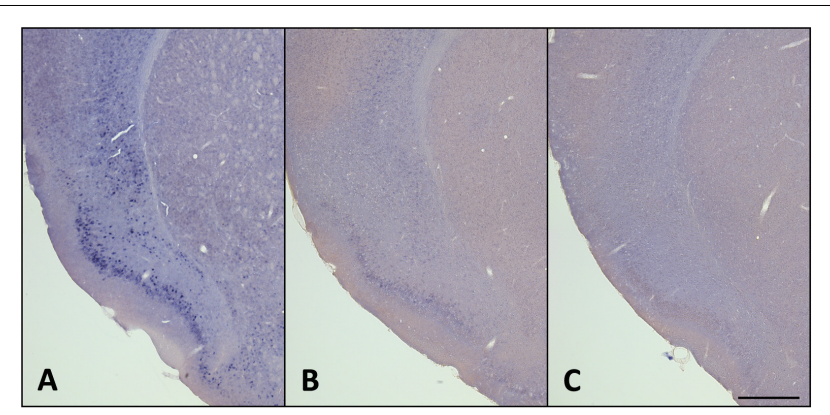

FIGURE 6 | Homer1a expression is reduced with recovery sleep (RS). (A) $3 \mathrm{~h}$ SD; (B) 3 h SD + 3 h RS; (C) SC. Bar size: $500 \mu \mathrm{m}$. See also Supplementary Table S1.

in these mice $(p=0.002)$ (Figure 7C and Supplementary Table S4); increases in $c$-fos with sleep deprivation based on qPCR were generally larger in the claustrum and piriform cortex. Similar data was observed by c-Fos immunostaining of these regions (Figure 5).
Further, c-fos expression levels were reduced with recovery sleep compared to values after sleep deprivation in all regions (Figure 7D, see Supplementary Table S4). Unlike results for homer $1 a$, where changes with sleep deprivation returned to levels seen in controls during sleep recovery, there remained residual increases in $c$-fos after sleep recovery compared to the 6-hour sleep control mice in all regions except the LC $(p=0.120)$. While results with recovery sleep were qualitatively similar across regions, there was again evidence of significant regional effects on the magnitude of the difference between recovery sleep and sleep loss $(p=0.0001)$ (Supplementary Table S4). Results are again supported by immunostaining of these regions (see Figure 5).

\section{Plasticity Marker Arc but Not BDNF Is Upregulated in Claustrum and Piriform Cortex With Sleep Deprivation}

We also examined the expression of the plasticity genes Arc and $B D N F$ by $\mathrm{q}-\mathrm{PCR}$ in the claustrum, piriform cortex, LC, TMN and lateral hypothalamus following $3 \mathrm{~h}$ of sleep deprivation and $3 \mathrm{~h}$ of recovery sleep (see Supplementary Table S5). Arc, like homerla, is increased in the claustrum and piriform
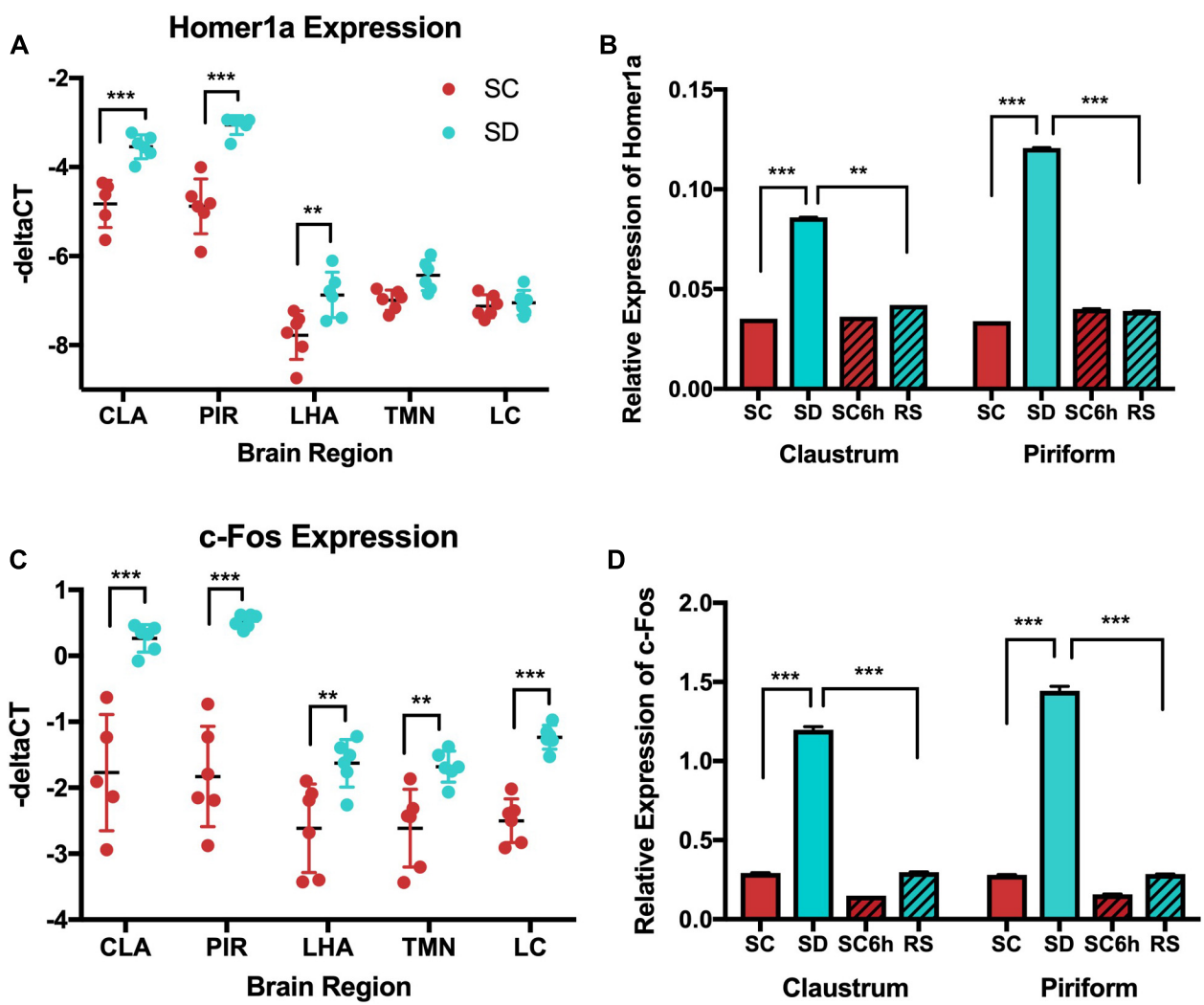

FIGURE 7 | QPCR shows increased homer1a expression in the claustrum (CLA) and piriform cortex (PIR) with sleep deprivation (SD) and c-fos increases in response to SD across all regions. (A) Homer1a qPCR across all brain regions in SC and SD conditions; (B) Homer1a qPCR across all behavioral conditions in claustrum and piriform. Homer1a expression is increased with SD in claustrum ( $p<0.001$ ) and piriform cortex ( $p<0.0001)$ SC (sleep control); SD (sleep deprived); RS (recovery sleep); LHA (lateral hypothalamus);PIR (piriform cortex); CLA (claustrum); TMN (Tuberomammillary nucleus); LC (Locus coeruleus). C-fos is increased in response to $\mathrm{SD}$ in all regions and is reduced with recovery sleep in the claustrum and piriform. C) qPCR of $c$-Fos shows increased $c$-Fos in response to SD in all regions tested ( 0 < 0.001); D) c-Fos qPCR across sleep deprivation (SD), 3 h sleep control (SC), 6 h diurnal sleep control (SC6h), and recovery sleep (RS) in young mice, $(p<0.001)$. See also Supplementary Figure S1 and Supplementary Tables S2-S4. 

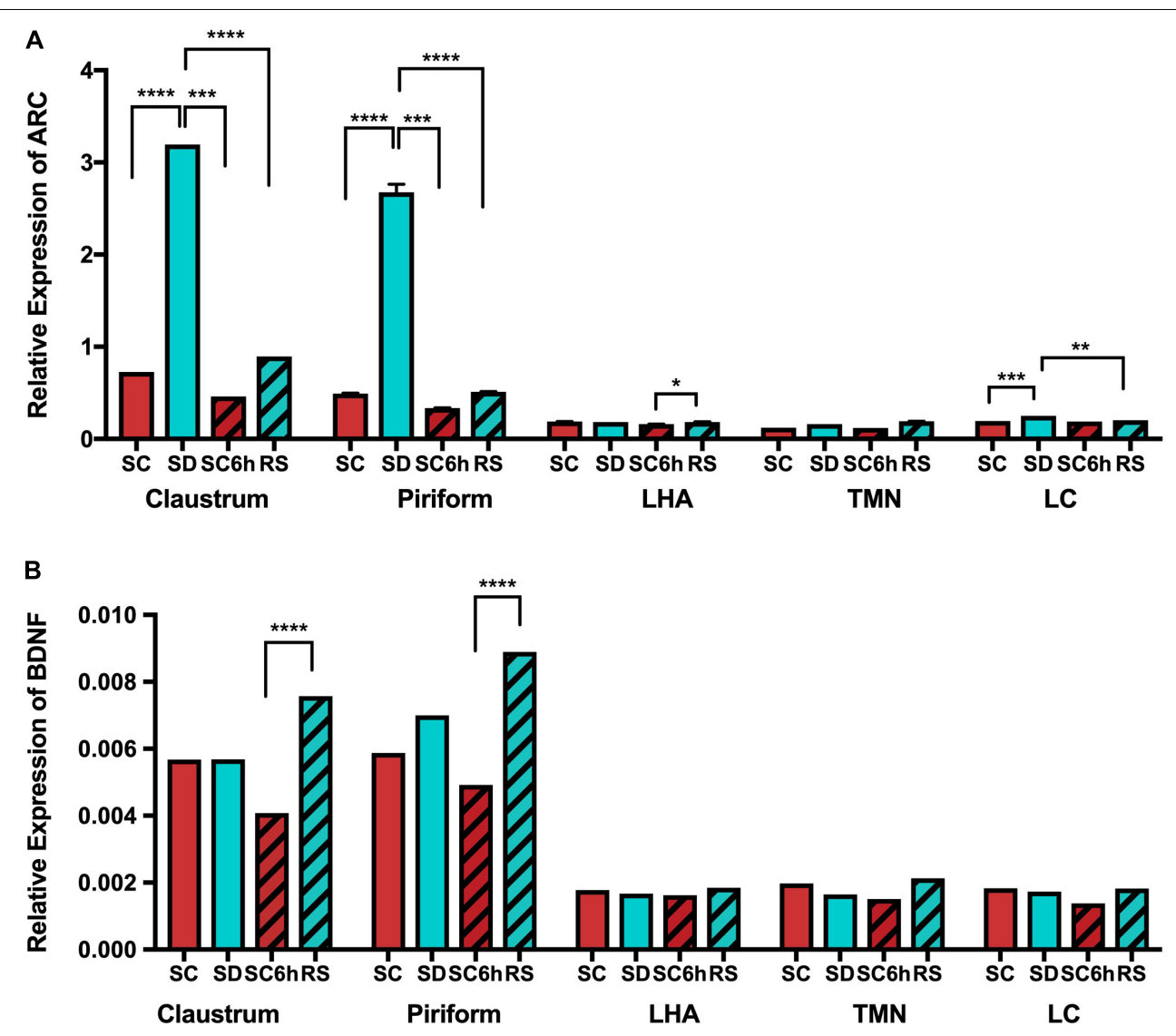

FIGURE 8 | QPCR shows increased Arc expression in claustrum and piriform cortex with SD. (A) Arc expression is shown across all conditions and brain regions. (B) BDNF expression is also shown across all brain regions and conditions.

cortex in mice subjected to $3 \mathrm{~h}$ of sleep deprivation when compared to undisturbed sleeping controls (all $p<0.0001$; Figure 8). Similarly, Arc values were significantly reduced with recovery sleep compared to sleep deprivation in these regions (all $p<0.0001$; Supplementary Table S5), although there remained residual increases $(p<0.009)$. Arc expression is not increased in the lateral hypothalamus or TMN with sleep deprivation, however, there were small, but significant increases in Arc transcript in the LC with sleep deprivation $(p=0.0046)$ that was reduced on average with recovery sleep (Supplementary Table S5 and Figure 8). We found no changes in BDNF transcript levels in any of the brain regions examined with sleep deprivation or recovery sleep when compared to undisturbed diurnal controls (Figure 8).

\section{DISCUSSION}

In this study, we examined the effect of acute sleep loss on homerla expression levels and spatial distribution in the brains of mice. The goal was to identify the early molecular changes that occur with sleep loss, particularly with homerla as an immediate early gene product, as opposed to the changes that occur with extended sleep loss, as described by Maret et al. (2007).
Intriguingly, we found that homerla expression increased in the claustrum, piriform, and cingulate cortices of these mice and to a much larger extent than in other brain regions. Importantly, homerla expression was low and changed little in classically studied wake-active neuronal groups following sleep deprivation. Our in situ hybridization results were confirmed with q-PCR. Namely, homerla expression increased in the claustrum, piriform cortex, and cingulate cortex following sleep deprivation. This increase was not present in classically studied wake-active regions, i.e., the tuberomammillary nucleus or the locus coeruleus. Further, following recovery sleep after sleep loss, homer1a expression levels were reduced in the claustrum and piriform cortex. QPCR data also demonstrated that homerla expression levels were reduced to the same extent with recovery sleep in both the claustrum and piriform cortex.

Homer 1 encodes constitutively expressed long-form proteins that serve as scaffolds in the post synapse, connecting important signaling molecules such as Shank proteins, mGluRs, and $\mathrm{IP}_{3}$ calcium receptors on the endoplasmic reticulum (Paschen and Mengesdorf, 2003; Piers et al., 2012). The short-form homerla, which lacks the C-terminal coiled-coiled domain, is a dominant negative that prevents dimerization and hence breaks the scaffold connections (Kammermeier and Worley, 2007). Its transcription is increased with neuronal activity (Bottai et al., 2002) and 
has been thought of as an immediate early gene. Our data indicate, however, that there is a dissociation between increases in c-Fos, which also occurs with neuronal activity (Hoffman et al., 1993) and increases in homerla expression. Some areas such as claustrum show increase in both whereas other neuronal groups (TMN, LC, and orexin neurons) show only increases in c-fos but not homerla.

Homerla is involved in important synaptic processes, specifically synaptic remodeling during sleep. It has been proposed that at sleep onset homerla protein is targeted to synapses where it binds mGluR1/5 and remodels or activates mGluR1/5 signaling to drive synaptic down-scaling (Diering et al., 2017). Previously, our work has shown that mice lacking homerla globally are unable to maintain wakefulness. Mice lacking homerla had short bouts of wakefulness and not the long bouts that occur in the early part of the lights off period (Naidoo et al., 2012). The neuronal basis of this effect on behavior is not known. The results presented here demonstrate that homer1a increases with extended wakefulness in the claustrum, as well and the piriform and cingulate cortices, which have not been previously implicated as key regulators of sleep and wake. The cingulate cortex, however, is known to play a role in emotional processing, learning and memory. Recent studies have revealed that abnormal connectivity in the anterior cingulate cortex is associated with insomnia (Yan et al., 2018). Further, the piriform is mostly involved in olfaction. It is known that during slowwave sleep, the piriform cortex becomes hypo-responsive to odor stimulation and functional connectivity to cortical regions is enhanced (Barnes and Wilson, 2014). In mice, it is unsurprising that both these regions demonstrate synaptic activity in the form of homerla expression, though the role of homerla expression in these regions is not exactly known and whether there is a role for homer in connectivity within these regions is unknown.

What we found particularly interesting were the changes in homer 1a expression levels in the claustrum, as very little is known about the function of this region. The claustrum is an enigmatic brain region whose function is unknown. The claustrum is a thin, irregular, laminar neuronal structure located beneath the inner surface of the neocortex, near the insula, with extensive reciprocal projections to numerous cortical and subcortical regions, such as the prefrontal cortex, primary sensory cortices, thalamus, and reticular formation (Smith and Alloway, 2010; Torgerson and Van Horn, 2014; Torgerson et al., 2015; Wang et al., 2017). Based on its structure and connectivity, Crick and Koch proposed that the claustrum integrates and binds together different cortical inputs, such as color, smell, sound, and touch, into a single unifying experience in consciousness (Crick and Koch, 2005). The claustrum is highly integrated with most cortical regions. Several qualitative studies indicate that the claustrum has topographical and reciprocal connections with the motor, premotor, orbitofrontal, prefrontal, parietal, cingulate, temporal, visual, perirhinal, and entorhinal cortices (Edelstein and Denaro, 2004; Druga et al., 2014; Zingg et al., 2014). As a result of these connections, it has been postulated to regulate conscious experiences (Crick and Koch, 2003, 2005; Blumenfeld, 2014). More recent quantitative studies using AAV tracers have confirmed that the claustrum has strong reciprocal and bilateral connections with prefrontal and cingulate areas as well as strong reciprocal connections with the ipsilateral temporal and retrohippocampal areas (Wang et al., 2017). The latter study suggests that the claustrum may play a crucial role in a variety of cognitive processes. Recent data indicate that several claustrum neurons wrap around the entire mouse brain (Koch, 2017), further emphasizing its connectivity to the whole brain. The claustrum has been hypothesized to be a saliency detector (Remedios et al., 2014; Smythies et al., 2014), identifying novel stimuli as well as a synchrony detector, integrating oscillations from other brain regions (Smythies et al., 2012). Case studies have shown that electrical stimulation of the claustrum results in a loss of consciousness (Koubeissi et al., 2014) and that claustrum damage is associated with the duration of loss of consciousness (Chau et al., 2015).

Importantly, the claustrum consists of a heterogeneous population of excitatory and inhibitory neurons (Kitanishi and Matsuo, 2017). Our data indicate that the increase in homer1a expression with extended wakefulness is specific to excitatory CAMKII $\alpha$-positive neurons. This suggests a potential mechanism to explain the role of homer $1 a$ in the maintenance of wakefulness. Because the claustrum is thought to be involved in synchronizing oscillations from the cortex (Smythies et al., 2014), it is possible that homerla expression in excitatory cells potentiates the development of gamma oscillations in the claustrum. Gamma oscillations are low amplitude, high frequency brain waves associated with wake and rapid eye movement (REM) sleep. Consistent with this idea, a recent study by Renouard et al. suggests that the claustrum may activate the cortex during REM sleep (Renouard et al., 2015). It is conceivable that increased homer1a expression in the claustrum strengthens gamma oscillations thereby producing a mechanism that sustains wakefulness. Further research manipulating claustrum neurons and homerla levels in these neurons is needed to understand whether gamma oscillations are altered and could regulate sleep and wake behavioral states. More recent data also suggest a role for the claustrum in insomnia, as genes found to be associated with insomnia using GWAS were also found to contain genes that are expressed in the claustrum (Jansen et al., 2019).

In conclusion, homer1a is specifically upregulated in the claustrum and piriform cortex, but not in classic wake-active neuronal groups. Recovery sleep following sleep deprivation reduces homer 1a expression in these brain regions back to control levels. Because homerla has many functions (Szumlinski et al., 2006; Leber et al., 2017) and binds to different intracellular signaling molecules, it is crucial to understand the role of homerla in regulating sleep-wake behaviors. Our results raise the possibility that the role of homerla in sustaining wakefulness is mediated by its effect on excitatory neurons in the claustrum.

\section{DATA AVAILABILITY STATEMENT}

The raw data supporting the conclusions of this manuscript will be made available by the authors, without undue reservation, to any qualified researcher. 


\section{ETHICS STATEMENT}

The methods and study protocols were approved in full by the Institutional Animal Care and Use Committee of the University of Pennsylvania and conformed to the revised National Institutes of Health Office of Laboratory Animal Welfare Policy.

\section{AUTHOR CONTRIBUTIONS}

NN designed the experiments, analyzed data, and wrote the manuscript. AP edited the manuscript. JZ and XG performed the experiments. JH performed the experiments, analyzed the data, and wrote the manuscript. BK conducted all the statistical analyses for the project.

\section{REFERENCES}

Bakker, R., Tiesinga, P., and Kotter, R. (2015). The scalable brain atlas: instant webbased access to public brain atlases and related content. Neuroinformatics 13, 353-366. doi: 10.1007/s12021-014-9258-x

Barnes, D. C., and Wilson, D. A. (2014). Sleep and olfactory cortical plasticity. Front. Behav. Neurosci. 8:134. doi: 10.3389/fnbeh.2014.00134

Blumenfeld, H. (2014). A master switch for consciousness? Epilepsy Behav. 37, 234-235. doi: 10.1016/j.yebeh.2014.07.008

Bottai, D., Guzowski, J. F., Schwarz, M. K., Kang, S. H., Xiao, B., Lanahan, A., et al. (2002). Synaptic activity-induced conversion of intronic to exonic sequence in Homer 1 immediate early gene expression. J. Neurosci. 22, 167-175. doi: 10.1523/jneurosci.22-01-00167.2002

Chau, A., Salazar, A. M., Krueger, F., Cristofori, I., and Grafman, J. (2015). The effect of claustrum lesions on human consciousness and recovery of function. Conscious. Cogn. 36, 256-264. doi: 10.1016/j.concog.2015. 06.017

Crick, F., and Koch, C. (2003). A framework for consciousness. Nat. Neurosci. 6, $119-126$.

Crick, F. C., and Koch, C. (2005). What is the function of the claustrum? Philos. Trans. R. Soc. Lond. B Biol. Sci. 360, 1271-1279. doi: 10.1098/rstb.2005. 1661

Diering, G. H., Nirujogi, R. S., Roth, R. H., Worley, P. F., Pandey, A., and Huganir, R. L. (2017). Homerla drives homeostatic scaling-down of excitatory synapses during sleep. Science 355, 511-515. doi: 10.1126/science.aai8355

Druga, R., Salaj, M., Barinka, F., Edelstein, L., and Kubova, H. (2014). Calretinin immunoreactivity in the claustrum of the rat. Front. Neuroanat. 8:160. doi: 10.3389/fnana.2014.00160

Edelstein, L. R., and Denaro, F. J. (2004). The claustrum: a historical review of its anatomy, physiology, cytochemistry and functional significance. Cell. Mol. Biol. (Noisy-le-grand) 50, 675-702.

Grabinski, T. M., Kneynsberg, A., Manfredsson, F. P., and Kanaan, N. M. (2015). A method for combining RNAscope in situ hybridization with immunohistochemistry in thick free-floating brain sections and primary neuronal cultures. PLoS One 10:e0120120. doi: 10.1371/journal.pone. 0120120

Hochberg, Y. (1988). A sharper Bonferroni procedure for multiple tests of significance. Biometrika 75, 800-802. doi: 10.1093/biomet/75.4.800

Hoffman, G. E., Lee, W. S., Smith, M. S., Abbud, R., Roberts, M. M., Robinson, A. G., et al. (1993). c-Fos and Fos-related antigens as markers for neuronal activity: perspectives from neuroendocrine systems. NIDA Res. Monogr. 125, $117-133$.

Hu, J. H., Park, J. M., Park, S., Xiao, B., Dehoff, M. H., Kim, S., et al. (2010). Homeostatic scaling requires group I mGluR activation mediated by Homerla. Neuron 68, 1128-1142. doi: 10.1016/j.neuron.2010.11.008

\section{FUNDING}

This work was funded by National Institutes of Health P01AG17628 and R56AG061057.

\section{ACKNOWLEDGMENTS}

The authors thank Dr. Dominique Eghlidi for assistance with figures and Dr. Paul Worley for helpful discussions.

\section{SUPPLEMENTARY MATERIAL}

The Supplementary Material for this article can be found online at: https://www.frontiersin.org/articles/10.3389/fnins. 2020.00188/full\#supplementary-material

Huang, Y., and Hsu, J. (1994). Hochberg's step-up method: cutting corners off Holm's step-down method. Biometrika 94, 965-975. doi: 10.1093/biomet/ asm067

Jansen, P. R., Watanabe, K., Stringer, S., Skene, N., Bryois, J., Hammerschlag, A. R., et al. (2019). Genome-wide analysis of insomnia in 1,331,010 individuals identifies new risk loci and functional pathways. Nat. Genet. 51, 394-403. doi: 10.1038/s41588-018-0333-3

Kammermeier, P. J., and Worley, P. F. (2007). Homer 1a uncouples metabotropic glutamate receptor 5 from postsynaptic effectors. Proc. Natl. Acad. Sci. U.S.A. 104, 6055-6060. doi: 10.1073/pnas.0608991104

Kitanishi, T., and Matsuo, N. (2017). Organization of the claustrum-to-entorhinal cortical connection in mice. J. Neurosci. 37, 269-280. doi: 10.1523/JNEUROSCI. $1360-16.2016$

Koch, C. (2017). A giant neuron found wrapped around entire mouse brain. Nature 543, 14-15. doi: 10.1038/nature.2017.21539

Koubeissi, M. Z., Bartolomei, F., Beltagy, A., and Picard, F. (2014). Electrical stimulation of a small brain area reversibly disrupts consciousness. Epilepsy Behav. 37, 32-35. doi: 10.1016/j.yebeh.2014.05.027

Leber, S. L., Llenos, I. C., Miller, C. L., Dulay, J. R., Haybaeck, J., and Weis, S. (2017). Homerla protein expression in schizophrenia, bipolar disorder, and major depression. J. Neural. Transm. 124, 1261-1273. doi: 10.1007/s00702-0171776-x

Lein, E. S., Hawrylycz, M. J., Ao, N., Ayres, M., Bensinger, A., Bernard, A., et al. (2007). Genome-wide atlas of gene expression in the adult mouse brain. Nature $445,168-176$.

Mackiewicz, M., Paigen, B., Naidoo, N., and Pack, A. I. (2008). Analysis of the QTL for sleep homeostasis in mice: homerla is a likely candidate. Physiol. Genomics 33, 91-99. doi: 10.1152/physiolgenomics.00189.2007

Mackiewicz, M., Shockley, K. R., Romer, M. A., Galante, R. J., Zimmerman, J. E., Naidoo, N., et al. (2007). Macromolecule biosynthesis: a key function of sleep. Physiol. Genomics 31, 441-457. doi: 10.1152/physiolgenomics.00275.2006

Maret, S., Dorsaz, S., Gurcel, L., Pradervand, S., Petit, B., Pfister, C., et al. (2007). Homerla is a core brain molecular correlate of sleep loss. Proc. Natl. Acad. Sci. U.S.A. 104, 20090-20095. doi: 10.1073/pnas.0710131104

Naidoo, N., Ferber, M., Galante, R. J., McShane, B., Hu, J. H., Zimmerman, J., et al. (2012). Role of homer proteins in the maintenance of sleep-wake states. PLoS One 7:e35174. doi: 10.1371/journal.pone.0035174

Naidoo, N., Ferber, M., Master, M., Zhu, Y., and Pack, A. I. (2008). Aging impairs the unfolded protein response to sleep deprivation and leads to proapoptotic signaling. J. Neurosci. 28, 6539-6548. doi: 10.1523/JNEUROSCI.5685-07.2008

Naidoo, N., Zhu, J., Zhu, Y., Fenik, P., Lian, J., Galante, R., et al. (2011). Endoplasmic reticulum stress in wake-active neurons progresses with aging. Aging Cell 10, 640-649. doi: 10.1111/j.1474-9726.2011.00699.x

Nelson, S. E., Duricka, D. L., Campbell, K., Churchill, L., and Krueger, J. M. (2004). Homerla and $1 b c$ levels in the rat somatosensory cortex vary with the time 
of day and sleep loss. Neurosci. Lett. 367, 105-108. doi: 10.1016/j.neulet.2004. 05.089

Palkovits, M. (1983). Punch sampling biopsy technique. Methods Enzymol. 103, 368-376. doi: 10.1016/s0076-6879(83)03025-6

Palkovits, M., Brownstein, M. J., and Vale, W. (1985). Distribution of corticotropin-releasing factor in rat brain. Fed. Proc. 44, 215-219.

Papadopulos, F., Spinelli, M., Valente, S., Foroni, L., Orrico, C., Alviano, F., et al. (2007). Common tasks in microscopic and ultrastructural image analysis using ImageJ. Ultrastruct. Pathol. 31, 401-407. doi: 10.1080/01913120701719189

Paschen, W., and Mengesdorf, T. (2003). Conditions associated with ER dysfunction activate homer la expression. J. Neurochem. 86, 1108-1115. doi: 10.1046/j.1471-4159.2003.01884.x

Piers, T. M., Kim, D. H., Kim, B. C., Regan, P., Whitcomb, D. J., and Cho, K. (2012). Translational concepts of mGluR5 in synaptic diseases of the brain. Front. Pharmacol. 3:199. doi: 10.3389/fphar.2012.00199

Remedios, R., Logothetis, N. K., and Kayser, C. (2014). A role of the claustrum in auditory scene analysis by reflecting sensory change. Front. Syst. Neurosci. 8:44. doi: $10.3389 /$ fnsys.2014.00044

Renouard, L., Billwiller, F., Ogawa, K., Clement, O., Camargo, N., Abdelkarim, M., et al. (2015). The supramammillary nucleus and the claustrum activate the cortex during REM sleep. Sci. Adv. 1:e1400177. doi: 10.1126/sciadv.140 0177

Schmittgen, T. D., and Livak, K. J. (2008). Analyzing real-time PCR data by the comparative CT method. Nat. Protoc. 3, 1101-1108. doi: 10.1038/nprot.2008.73

Smith, J. B., and Alloway, K. D. (2010). Functional specificity of claustrum connections in the rat: interhemispheric communication between specific parts of motor cortex. J. Neurosci. 30, 16832-16844. doi: 10.1523/JNEUROSCI.443810.2010

Smythies, J., Edelstein, L., and Ramachandran, V. (2012). Hypotheses relating to the function of the claustrum. Front. Integr. Neurosci. 6:53. doi: 10.3389/fnint. 2012.00053

Smythies, J., Edelstein, L., and Ramachandran, V. (2014). Hypotheses relating to the function of the claustrum II: does the claustrum use frequency codes? Front. Integr. Neurosci. 8:7. doi: 10.3389/fnint.2014.00007
Szumlinski, K. K., Kalivas, P. W., and Worley, P. F. (2006). Homer proteins: implications for neuropsychiatric disorders. Curr. Opin. Neurobiol. 16, 251-257. doi: 10.1016/j.conb.2006.05.002

Torgerson, C. M., Irimia, A., Goh, S. Y., and Van Horn, J. D. (2015). The DTI connectivity of the human claustrum. Hum. Brain Mapp. 36, 827-838. doi: 10.1002/hbm.22667

Torgerson, C. M., and Van Horn, J. D. (2014). A case study in connectomics: the history, mapping, and connectivity of the claustrum. Front. Neuroinform. 8:83. doi: 10.3389/fninf.2014.00083

Vandesompele, J., De Preter, K., Pattyn, F., Poppe, B., Van Roy, N., De Paepe, A., et al. (2002). Accurate normalization of real-time quantitative RT-PCR data by geometric averaging of multiple internal control genes. Genome Biol. 3:research0034.

Wang, Q., Ng, L., Harris, J. A., Feng, D., Li, Y., Royall, J. J., et al. (2017). Organization of the connections between claustrum and cortex in the mouse. J. Comp. Neurol. 525, 1317-1346. doi: 10.1002/cne.24047

Yan, C. Q., Liu, C. Z., Wang, X., Huo, J. W., Zhou, P., Zhang, S., et al. (2018). Abnormal functional connectivity of anterior cingulate cortex in patients with primary insomnia: a resting-state functional magnetic resonance imaging study. Front. Aging Neurosci. 10:167. doi: 10.3389/fnagi.2018.00167

Zingg, B., Hintiryan, H., Gou, L., Song, M. Y., Bay, M., Bienkowski, M. S., et al. (2014). Neural networks of the mouse neocortex. Cell 156, 1096-1111. doi: 10.1016/j.cell.2014.02.023

Conflict of Interest: The authors declare that the research was conducted in the absence of any commercial or financial relationships that could be construed as a potential conflict of interest.

Copyright (c) 2020 Zhu, Hafycz, Keenan, Guo, Pack and Naidoo. This is an openaccess article distributed under the terms of the Creative Commons Attribution License (CC BY). The use, distribution or reproduction in other forums is permitted, provided the original author(s) and the copyright owner(s) are credited and that the original publication in this journal is cited, in accordance with accepted academic practice. No use, distribution or reproduction is permitted which does not comply with these terms. 